Acta Botanica Mexicana 69: 115-131 (2004)

\title{
EL GÉNERO HELIOPSIS (HELIANTHEAE; ASTERACEAE) EN MÉXICO Y LAS ALCAMIDAS PRESENTES EN SUS RAÍCES
}

\author{
Abraham García-Chávez, Enrique Ramírez Chávez y Jorge Molina-Torres \\ Laboratorio de Fitobioquímica, Departamento de Biotecnología y Bioquímica, \\ CINVESTAV-IPN Unidad Irapuato, km 9.6 Libramiento Norte, \\ Apdo. postal 629, 36500 Irapuato, Guanajuato, México
}

\begin{abstract}
RESUMEN
El género Heliopsis pertenece a la tribu Heliantheae de la familia Asteraceae. Incluye 14 especies, la mayoría endémicas a México y aún no todas totalmente definidas. Una de ellas, Heliopsis longipes, utilizada en la medicina tradicional, se ha caracterizado por el alto contenido de alcamidas en sus raíces. El estudio de tales metabolitos ha derivado en una serie de investigaciones sobre su presencia en otros representantes del género Heliopsis de México. El escrito sintetiza y actualiza la información bibliográfica referente a la taxonomía del género y se presentan los resultados obtenidos del análisis cuantitativo y cualitativo de las alcamidas detectadas en raíces de seis especies. Se discute la importancia quimiotaxonómica de estos metabolitos en el mismo género.
\end{abstract}

Palabras clave: alcamidas, Asteraceae, Heliantheae, Heliopsis, México.

\begin{abstract}
The genus Heliopsis belongs to the tribe Heliantheae in the Asteraceae family. It includes 14 species, most of them endemic to Mexico. One of the species, Heliopsis longipes, is utilized in traditional medicine. The high level of alkamides in its roots has already been chemically characterized. The study of these metabolites is displayed in a comparative analysis on the presence of alkamides in six Mexican species of Heliopsis. The quantitative and qualitative analysis of the alkamides stored in roots of each species is presented. Chemotaxonomic importance of alkamides in this genus is also discussed. In this work we review and update the bibliography referring to the taxonomy of the genus.
\end{abstract}

Key words: alkamides, Asteraceae, Heliantheae, Heliopsis, Mexico. 


\section{ASPECTOS BOTÁNICOS}

Datos generales relativos a Heliopsis

El género Heliopsis pertenece a la tribu Heliantheae de la familia Asteraceae. La mencionada tribu comprende cerca de 2,500 especies agrupadas en 189 géneros y está fraccionada en diez subtribus. Heliopsis, asociado Zinnia, Philactis, Sanvitalia, Acmella, Podachaenium, Squamopappus, Spilanthes y Salmea, conforma la subtribu Zinniinae (Bremer, 1994), cuyos miembros comparten, en su morfología, la presencia de capítulos solitarios, receptáculos cónicos o hemisféricos, flores liguladas pistiladas, fértiles y persistentes, flores del disco fértiles y aquenios del disco lateralmente comprimidos o angulados. La mayor diferencia que separa las especies de Heliopsis de las de otros géneros es la siguiente combinación de caracteres: la presencia de flores fértiles en el disco, flores liguladas persistentes y fértiles, corolas sésiles y persistentes, aquenios gruesos, 3 a 4-angulares y vilano ausente.

Aunque restringida al hemisferio occidental, la distribución de este género es amplia, abarcando desde la región de las grandes planicies y el oriente de Estados Unidos, hasta Bolivia. Los hábitats donde viven sus especies son variados, desde regiones áridas hasta las francamente húmedas y desde las cálidas hasta las de clima templado; la abundancia de varias es favorecida por el disturbio y al menos una se comporta como maleza. Está representado por 14 especies conocidas, aunque tal número varía con el criterio de los diferentes autores. De estas especies, diez se encuentran en nuestro país y ocho lo son de manera endémica (Cuadro 1).

Los estudios taxonómicos realizados sobre Heliopsis han sido escasos, lo que ha provocado inseguridad en la interpretación de algunos de sus taxa. Dichos estudios se han basado en las características morfológicas. Las especies de este género son plantas herbáceas perennes o anuales; hojas opuestas o a veces alternas, subenteras a dentadas; cabezuelas terminales o axilares; involucro hemisférico a anchamente campanulado, sus brácteas de tamaño subigual, las exteriores a menudo herbáceas; receptáculo convexo a cónico, provisto de páleas persistentes; flores liguladas fértiles, sus corolas sésiles (carentes de tubo), persistentes, amarillas, rojas o moradas; flores del disco hermafroditas aunque a veces mayormente estériles, sus corolas tubulosas, amarillas, amarillo-cafés o moradas; anteras con las bases brevemente aflechadas; ramas del estilo de las flores hermafroditas lineares, aplanadas, provistas de apéndice corto; aquenios gruesos, 3 a 4-angulares, vilano ausente (Rzedowski, Rzedowski et al., 2001). 
La mayor parte de los representantes de Heliopsis son plantas perennes con la excepción de cinco especies anuales (H. annua, $H$. anomala, H. filifolia, $H$. parviceps y $H$. sinaloensis) endémicas a México. Las perennes presentan raíces gruesas y carnosas divididas o sin dividir.

Cuadro 1. Especies conocidas de Heliopsis.

\begin{tabular}{|l|l|}
\hline \multicolumn{1}{|c|}{ Especie } & \multicolumn{1}{|c|}{ Distribución Geográfica } \\
\hline De distribución restringida & $\begin{array}{l}\text { Localizadas en un área geográfica reducida, se encuentran } \\
\text { delimitadas por sistemas orográficos aislados, como lo son } \\
\text { pequeñas serranías, mesetas o valles aislados e incluso } \\
\text { cuencas endorreicas }\end{array}$ \\
\hline H. filifolia S. Wats. & Cuatro Ciénegas, Carneros y Puerto Colorado, Coahuila \\
\hline H. longipes (Gray) Blake & $\begin{array}{l}\text { Sierra Gorda, en el centro del país en la región de } \\
\text { colindancia de los estados de Guanajuato, San Luis Potosí } \\
\text { y Querétaro }\end{array}$ \\
\hline H. sinaloensis B. L. Turner & Imala, Sinaloa \\
\hline De distribución regional & $\begin{array}{l}\text { Localizadas a lo largo de varios estados del país, pero } \\
\text { sin exceder los límites nacionales }\end{array}$ \\
\hline H. annua Hemsl. & $\begin{array}{l}\text { Altiplano Mexicano principalmente en la región del } \\
\text { Bajío. Jalisco, Guanajuato, Querétaro, Michoacán, Estado } \\
\text { de México, Distrito Federal, Morelos, Tlaxcala, Puebla, } \\
\text { Hidalgo, San Luis Potosí, Zacatecas, Aguascalientes, } \\
\text { Durango, Chihuahua y Coahuila }\end{array}$ \\
\hline $\begin{array}{l}\text { H. anomala } \text { (M. E. Jones) B. } \\
\text { L. Turner }\end{array}$ & $\begin{array}{l}\text { Región de montañas y cañones costeros del Golfo de } \\
\text { California (Baja California, Baja California Sur y Sonora) }\end{array}$ \\
\hline $\begin{array}{l}\text { H. novogaliciana } \text { B. L. } \\
\text { Turner }\end{array}$ & $\begin{array}{l}\text { Sierra Madre Occidental. Jalisco, Nayarit, Sinaloa, } \\
\text { Chihuahua y Durango }\end{array}$ \\
\hline H. parviceps Blake & $\begin{array}{l}\text { Vertiente pacífica de México. Sinaloa, Michoacán, } \\
\text { Guerrero, Estado de México }\end{array}$ \\
\hline H. procumbens Hemsl. & $\begin{array}{l}\text { Eje Volcánico Transversal. Jalisco, Michoacán, Estado } \\
\text { de México, Distrito Federal, Hidalgo, Morelos, Tlaxcala, } \\
\text { Puebla y Veracruz }\end{array}$ \\
\hline
\end{tabular}


Cuadro 1. Continuación

\begin{tabular}{|l|l|}
\hline \multicolumn{1}{|c|}{ Especie } & \multicolumn{1}{c|}{ Distribución Geográfica } \\
\hline De distribución amplia & $\begin{array}{l}\text { La distribución de estas especies excede los límites } \\
\text { nacionales }\end{array}$ \\
\hline $\begin{array}{l}\text { H. buphthalmoides (Jacq.) } \\
\text { Dunal }\end{array}$ & $\begin{array}{l}\text { La especie de más amplia distribución geográfica del } \\
\text { género. Sierra Madre Occidental, Sierra Madre del Sur, } \\
\text { Cordillera Centroamericana y los Andes. Durango, } \\
\text { Sinaloa, Nayarit, Jalisco, Michoacán, Estado de México, } \\
\text { Morelos, Guerrero, Puebla, Oaxaca y Chiapas en México; } \\
\text { toda Centroamérica, Colombia, Venezuela, Perú y Bolivia } \\
\text { en Sudamérica }\end{array}$ \\
\hline H. parviflora Gray & $\begin{array}{l}\text { Zonas áridas y semiáridas del norte de México y sur de } \\
\text { Estados Unidos. Zacatecas, Aguascalientes, Durango, } \\
\text { Nuevo León, Coahuila, Chihuahua y Sonora en México; } \\
\text { California, Arizona, Nuevo México y Texas en los } \\
\text { Estados Unidos }\end{array}$ \\
\hline Extraterritoriales a México & Distribuidas solamente fuera de México \\
\hline H. decumbens Blake & Cajamaraca, Perú. Conocida sólo de la localidad del tipo \\
\hline H. gracilis (Gray) Nutt. & $\begin{array}{l}\text { Del suroeste de Georgia, norte y centro de Florida al } \\
\text { sur de Alabama }\end{array}$ \\
\hline H. helianthoides (L.) Sweet & Oriente de Estados Unidos \\
\hline H. lanceolata Blake & $\begin{array}{l}\text { Santander, Colombia. Conocida solo de la localidad del } \\
\text { tipo }\end{array}$ \\
\hline
\end{tabular}

En la Lámina 1 se puede observar que las hojas de Heliopsis presentan forma deltoidea, lanceolada, ovada o lanceo-ovada y caso especial es la filiforme, específica de $H$. filifolia. El margen foliar es una característica importante. La mayoría de las especies presentan hojas dentadas o semidentadas, aunque algunas las tienen casi enteras o irregularmente crenadas. La textura es generalmente firme, con excepción de las plantas anuales que tienen hojas usualmente delgadas y frágiles. El pecíolo ha sido utilizado como un carácter valioso en la separación de las subespecies de H. helianthoides, tanto por su longitud como por su forma (Fisher, 1957).

Para el caso del involucro, sus brácteas son de poco valor para la separación de los taxa, ya que son altamente variables dentro de una especie dada, con respecto 

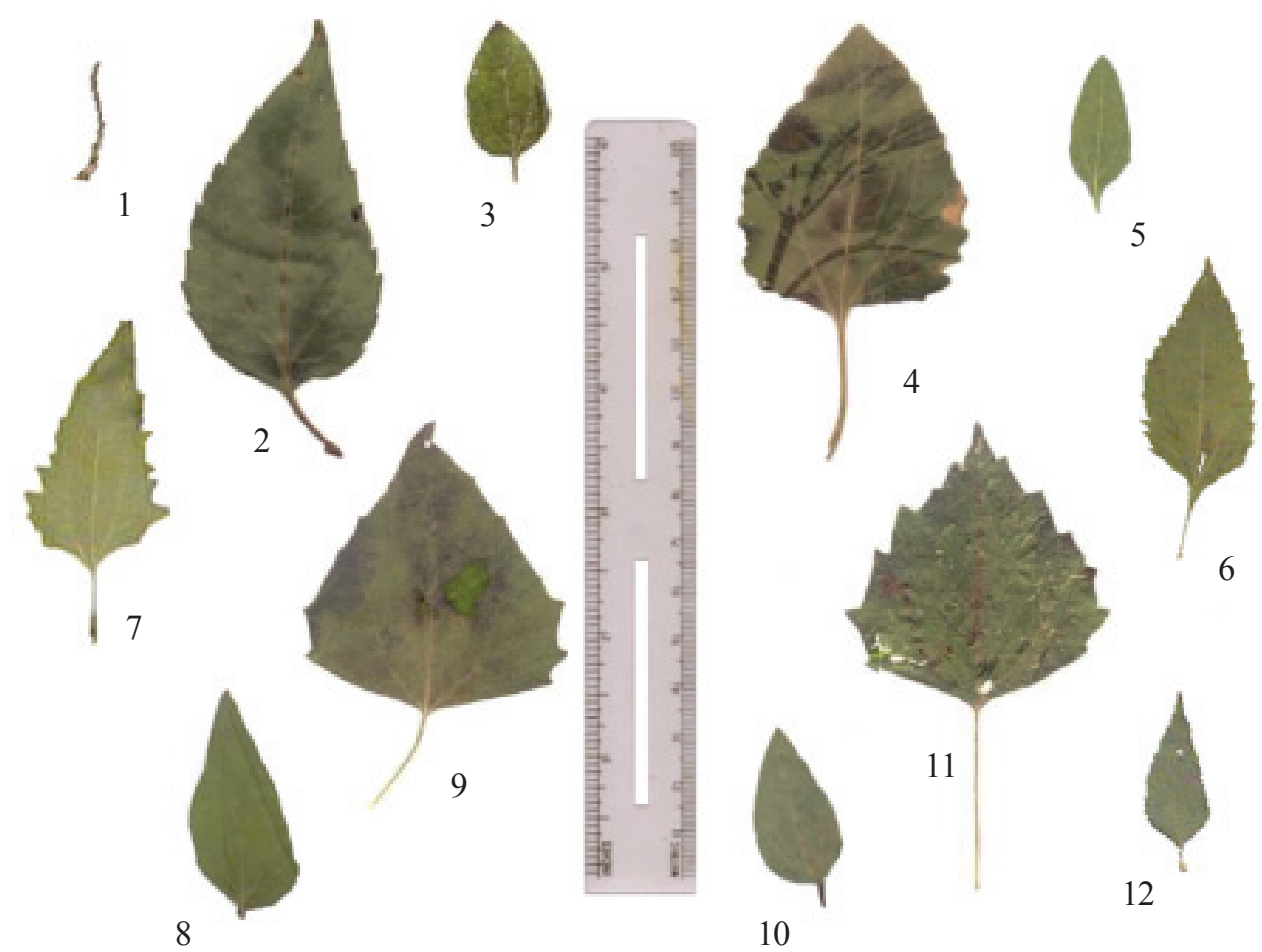

Lámina 1. Hojas de algunas especies del género Heliopsis. Las hojas pertenecientes a las especies $H$. filifolia, $H$. helianthoides y $H$. gracilis son imagenes obtenidas de ejemplares depositados en el herbario de la Universidad de Texas, en Austin; las hojas pertenecientes a las especies $H$. parviceps y $H$. parvifolia, son imágenes obtenidas de ejemplares depositados en el herbario del Instituto de Ecología A. C. en Pátzcuaro, Michoacán. El resto de las hojas son imágenes de muestras colectadas por los autores. 1. H. filifolia. 2. H. buphthalmoides. 3. H. procumbens. 4. H. annua. 5. H. parviceps. 6. H. helianthoides. 7. H. parvifolia. 8. H. novogaliciana. 9. H. anomala. 10. H. longipes. 11. H. sinaloensis. 12. H. gracilis.

a la forma, tamaño y pubescencia. Cuando el involucro es de 2 ó 3 series, sus brácteas con frecuencia son foliáceas, tanto en las plantas de Estados Unidos como en las de México.

El receptáculo es en general convexo a cónico. Las páleas son lanceoladas, agudas y persistentes después de la madurez, con ápice de color rojo, púrpura o amarillo-café, características que en conjunto ofrecen buen criterio de separación de ciertos taxa. La forma, textura y tamaño de los aquenios es otra ayuda valiosa en la diferenciación de especies, como lo muestra la Lámina 2. 

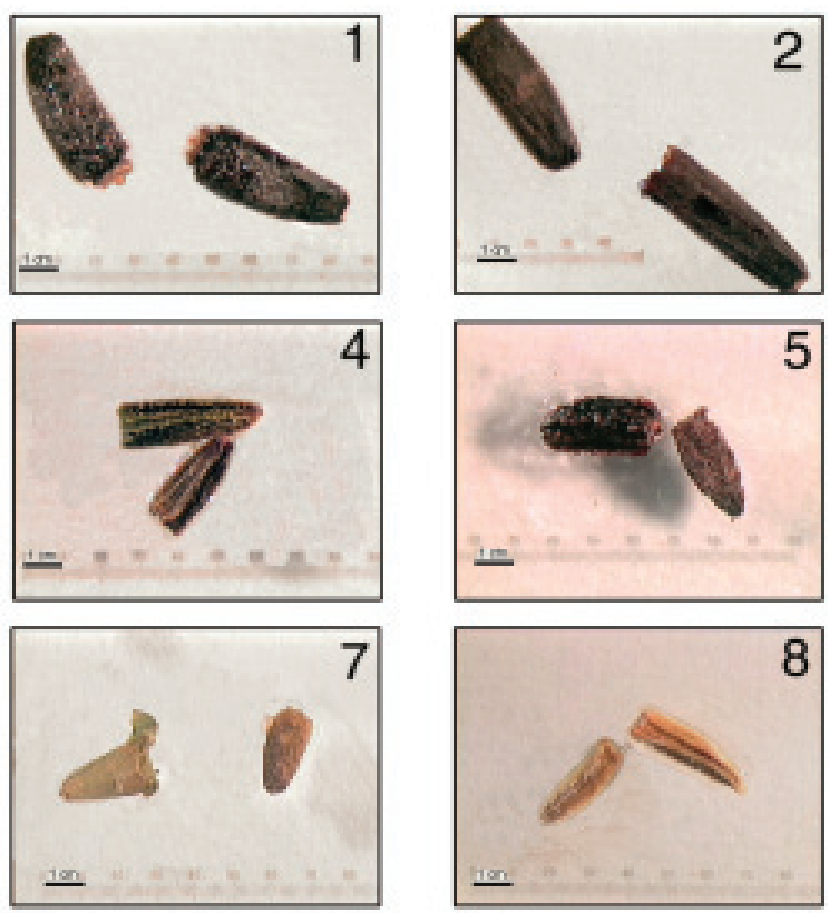
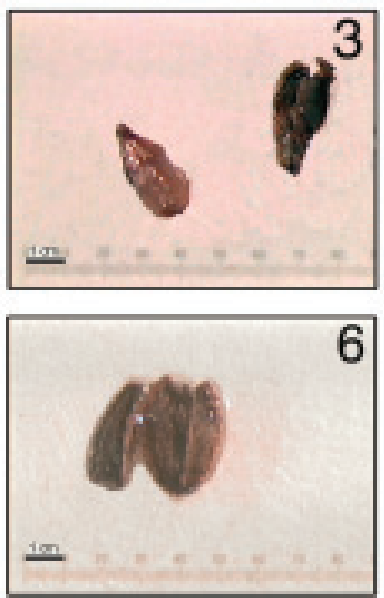

Lámina 2. Aquenios de algunas especies del género Heliopsis. Las imágenes mostradas son fotografías de materiales colectados por los autores y citados en el texto. 1. H. annua. 2. H. anomala. 3. H. aff. novogaliciana localidad Chavarría. 4. H. longipes localidad Puerto de Tablas. 5. H. longipes localidad San Cristóbal. 6. H. novogaliciana. 7. H. buphthalmoides. 8. H. procumbens. 9. H. sinaloensis.

Usos de las especies del género Heliopsis

La utilidad de estas plantas desde el punto de vista antropocéntrico se ha limitado a dos especies: Heliopsis helianthoides, comercializada en Estados Unidos como flor de ornato en jardinería bajo el nombre de "falso girasol" y Heliopsis longipes con aplicaciones más diversas en México, conocida comúnmente como chilcuague. Esta última tiene una larga tradición en la herbolaria indígena como lo indican sus denominaciones de origen náhuatl. Algunos nombres registrados son: ichcha, citado en la traducción de la obra de Francisco Hernández "De historia plantarum Novae Hispaniae"; chilcuán, significando chile de víbora; chilmécatl, de chili, chile y mécatl, mecate, aludiendo a las raíces filiformes y al sabor picante de éstas (Martínez, 1994); chilicuau, nombre que se usa en el municipio de San Joaquín, en Querétaro. Asimismo, Martínez (1994) registra pelitre o peritre como epítetos que 
probablemente se le asignaron durante la era de la colonia. En la actualidad se puede encontrar esta especie comercializada en los puestos de hierbas medicinales de casi todo el país con nombres más modernos como raíz de oro y raíz azteca.

Aunque la planta no se ha localizado en registros precolombinos, puede encontrarse en la literatura de la época colonial temprana, por ejemplo en los extractos de la obra de Francisco Hernández, "Quatro libros de la natvraleza" editada por Ximénez (1615). Más recientemente, Noriega (1902) en su "Curso de historia de drogas" la menciona en relación al reemplazo del peritre de África: “... Se suele sustituir con la raíz del chilcuam o peritre del país. Erygeron affinis (sic), que abunda en los alrededores de México y es probablemente la misma raíz abundante en la Sierra de Querétaro, conocida con el nombre de chilcuau" (sic). Es probable que esta incertidumbre esté relacionada con el error que se cometió en la identificación de las raíces cuando Acree y colaboradores aislaron por primera vez la afinina, como se menciona más adelante.

Por sus propiedades insecticidas, fue una de las plantas descubiertas por los Estados Unidos durante la segunda guerra mundial, observando que los extractos de estas raíces tienen el mismo grado de acción paralítica y toxicidad contra moscas y otros insectos, que el piretro (Little, 1948). Consciente de su utilidad como insecticida y su limitada disponibilidad, el Departamento de Agricultura de Estados Unidos se involucró en la búsqueda de otras especies del mismo género dentro de su territorio. Así de Heliopsis scabra Dunal se aisló la escabrina, una alcamida insecticida presente en sus raíces (Jacobson, 1951). Sin embargo, en ensayos preliminares se observó que la escabrina, caracterizada por múltiples ligaduras insaturadas y siendo más efectiva que las piretrinas en ensayos con mosca casera, mostró una alta toxicidad para mamíferos (Roark, 1951). Se desconoce qué especie se utilizó en realidad en estos estudios, ya que ese binomio actualmente está en desuso. Los ejemplares originalmente etiquetados como $H$. scabra, encontrados en el herbario de la Universidad de Texas en Austin, han sido reubicados, algunos como $H$. parvifolia y otros como $H$. helianthoides (observación personal) y en la actualidad solamente se reconoce la subespecie $H$. helianthoides ssp. scabra (Fisher, 1957). En consecuencia la estructura de la escabrina $N$-isobutil-1,3,7,9,14octadodecapentadienamida no ha podido ser confirmada, ni resuelta la isomería de sus ligaduras por no haberse aislado nuevamente.

Por fortuna el chilcuague no se extinguió por la euforia de su exportación, a pesar de que la población silvestre se vio severamente reducida. De acuerdo con Little (1948), el sitio de la colecta original del chilcuague fue el área sur y suroeste de la Sierra de Álvarez en San Luis Potosí. El mismo autor la localizó en la parte noroeste de Guanajuato en la Sierra Gorda, cerca de la carretera desde San Luis de la Paz en dirección oriental a Xichú, hasta Santa Catarina y en la región norte de Querétaro. A más de 50 años después de este informe, la distribución conocida 
es más o menos similar. En estas regiones de Guanajuato y de Querétaro se ha iniciado el cultivo del chilcuague que se lleva a cabo en forma limitada en las orillas de las parcelas o en la sombra de algunos árboles, en lugares protegidos del pastoreo, aunque a la fecha ya es posible observar terrenos dedicados completamente a plantíos de esta especie. Las personas que lo cultivan indican que a partir de la propagación por esqueje, y en algunos casos por semilla, el desarrollo total de las raíces transcurre en dos a tres años. Existe en la actualidad un esfuerzo del gobierno por el establecimiento de cultivos de chilcuague para garantizar los ingresos de la población en las regiones mencionadas. El precio de esta raíz puede ser satisfactorio para el productor, pero no existe un mercado suficientemente grande y estable fuera de la región de producción.

Conocimiento taxonómico del género Heliopsis

Fisher (1957) hizo una relación detallada de la evolución del conocimiento del género Heliopsis y como resultado de su revisión reconoció los siguientes taxa: $H$. annua Hemsl., H. brachactis Standl. ex T. R. Fisher, H. buphthalmoides (Jacq.) Dunal, $H$. decumbens Blake, $H$. filifolia S. Wats., H. gracilis (A. Gray) Nutt., $H$. helianthoides (L.) Sweet ssp. helianthoides, H. helianthoides (L.) Sweet ssp. occidentalis T. R. Fisher, H. lanceolata Blake, H. longipes (A. Gray) Blake, $H$. parviceps Blake, H. parvifolia A. Gray, H. procumbens Hemsl. y H. rubra T. R. Fisher. Más tarde, en 1961, Fisher adicionó una especie más al describir a $H$. pulchra, misma que es reconocida posteriormente como parte de $H$. buphthalmoides (McVaugh, 1984).

En 1958 Wilson describió a $H$. purpurea, con base en colectas de Hinton en Temascatepec, Estado de México, y en Cutzamala, Guerrero. De acuerdo con McVaugh (1984), H. purpurea es sinónimo de H. parviceps, ya que las pocas colecciones observadas con disco de color púrpura no presentan diferencias significativas fuera del color amarillo o púrpura de los radios.

Turner (1987) describió a $H$. novogaliciana, diferenciándola de $H$. procumbens principalmente por los caracteres de las hojas, los apéndices de las anteras amarillas y su distribución geográfica. En el mismo trabajo discutió las diferencias entre $H$. brachactis y $H$. sinaloensis, esta última también reconocida como especie nueva. En el siguiente año el mismo Turner (1988) propuso reemplazar el nombre de Heliopsis rubra T. R. Fisher por el de H. anomala (Jones) B. L. Turner.

Por último, Díaz-Piedrahíta (1990) estableció el binomio de H. oppositifolia (Lamarck) S. Díaz-Piedrahíta para substituir a H. buphthalmoides. Sin embargo, cabe hacer notar que Heliopsis oppositifolia (L.) Druce, nombre publicado en 1914 y con base en Rudbeckia oppositifolia (L.), tiene prioridad sobre Heliopsis 
oppositifolia (Lamarck) S. Díaz-Piedrahíta. Este último, al ser homónimo posterior, es ilegítimo de acuerdo con el Código Internacional de Nomenclatura Botánica (ICBN) y no debe usarse. En el presente trabajo, bajo tal consideración, conservamos el epíteto de $H$. buphthalmoides (Jacq.) Dun.

\section{ASPECTOS QUÍMICOS}

Alcamidas en el género Heliopsis

La condensación química de un ácido con una amina resulta en la formación de una amida. El grupo funcional amida es ubicuo, se encuentra en todos los organismos vivos constituyendo los enlaces peptídicos, esto es, la unión entre los aminoácidos para la formación de la estructura primaria de las proteínas, moléculas que son la base de la vida como la conocemos hasta ahora. Las amidas como metabolitos secundarios, por otra parte, no son tan frecuentes. Un ejemplo interesante de este grupo de compuestos es el de las alquilamidas o alcamidas que comprenden un conjunto de aproximadamente 70 estructuras conocidas, distribuidas en diversos niveles del Reino Vegetal.

Las alcamidas representan una clase singular de productos naturales que, desde el punto de vista biogénico, se forman al combinarse dos diferentes rutas metabólicas. La parte acídica se origina de un ácido graso de longitud de cadena de mediana a larga, que puede ser de ocho a dieciocho carbonos, generalmente alifática o lineal; éste, al condensarse con un aminoácido y en descarboxilación concomitante, resulta en la producción de una alcamida. Las amidas cuya cadena acídica es alifática, dependiendo del tipo de enlaces insaturados que presenten, se pueden separar en dos grupos: las alcamidas olefínicas, con al menos una doble ligadura; y las alcamidas acetilénicas, con al menos una triple ligadura (Greger, 1984). En el género Heliopsis sólo se han encontrado alcamidas alifáticas y acetilénicas. Otro conjunto lo constituyen las amidas que presentan anillos homo o heterocíclicos. Este tipo de estructuras se observan en forma particularmente diversa en la familia Piperaceae e incluye varios alcaloides (Parmar et al., 1997). Las alcamidas son consideradas compuestos bioactivos, esto es, una pequeña cantidad del compuesto evoca una respuesta notable en las células receptoras.

H. longipes fue la primera especie en la que se determinó la presencia de una alcamida olefínica (Acree et al., 1945). Sin embargo, la planta cuya muestra de raíces fue sometida para su análisis en laboratorio resultó erróneamente identificada como Erigeron affinis y así, la amida aislada fue denominada afinina. El descubrimiento de la afinina, un compuesto insecticida, en esta especie despertó interés por su notoria actividad. La afinina es la alcamida mayoritaria en las raíces 
de esta planta y principal responsable de los efectos biológicos específicos observados, entre los que se pueden considerar la acción de anestésico local, el estímulo organoléptico, así como la actividad insecticida y bactericida. Por mucho tiempo se consideró a la afinina como único componente bioactivo en este tejido, cuyo extracto crudo se ha utilizado para el estudio de sus aplicaciones, mismas que recientemente se han extendido (Romero et al., 1989; Gutiérrez-Lugo et al., 1996) mostrando un potencial agronómico importante en el control biológico de bacterias y hongos fitopatógenos (Ramírez-Chávez et al., 2000). El análisis fitoquímico más detallado de las raíces de $H$. longipes ha revelado la presencia de otros principios bioactivos y de propiedades antimicrobianas de algunas alcamidas individuales (Molina-Torres et al., 1995, 1996,1999).

Las alcamidas, por su actividad bactericida y fungicida, deben constituir un elemento de defensa en especies vegetales pertenecientes a grupos taxonómicos muy distantes. Estos metabolitos constitutivos o anticipinas se han encontrado hasta la fecha en diez familias: Aristolochiaceae, Asteraceae, Brassicaceae, Convolvulaceae, Euphorbiaceae, Menispermaceae, Piperaceae, Poaceae, Rutaceae y Solanaceae. En la subtribu Zinniinae de la familia Asteraceae presentan alcamidas los géneros Heliopsis, Acmella, Sanvitalia y Salmea (Greger, 1984; Christensen y Lam, 1991; Christensen, 1992) y su estudio puede revelar importancia quimiotaxonómica. En general las especies en que se han encontrado alcamidas frecuentemente han sido utilizadas por el hombre como plantas medicinales.

Conociendo que $H$. longipes contiene otras alcamidas, además de la afinina como mayoritaria, se realizó una exploración para analizar su existencia en otras especies del género Heliopsis y estimar si tiene significado taxonómico la presencia de estos metabolitos. De 1998 a 2001, se realizaron colectas del género Heliopsis en diversas regiones de México, incluyendo algunas localidades de la Sierra Madre Occidental no mencionadas anteriormente para esta género.

\section{Métodos}

Se colectaron especímenes del género Heliopsis correspondientes a siete especies en diez localidades. Los sitios de colecta, la fecha y el registro de herbario de las especies estudiadas se presentan en el Cuadro 2. Los especímenes colectados se depositaron en el Herbario del Instituto de Ecología A.C. en Pátzcuaro, Michoacán, con los registros citados en el Cuadro 2.

Para el análisis cualitativo y cuantitativo de las alcamidas se utilizó solamente la raíz. De cada espécimen se tomó una muestra de 1 gr de tejido fresco que fue congelada en nitrógeno líquido; se homogenizó perfectamente en mortero y se le adicionó $5 \mathrm{ml}$ de etanol absoluto. Cada muestra se sometió a sonicación durante 30 minutos a temperatura ambiente; se centrifugó a 3,000 rpm por 5 minutos y después 
García-Chávez et al.: Heliopsis en México y las alcamidas en sus raíces

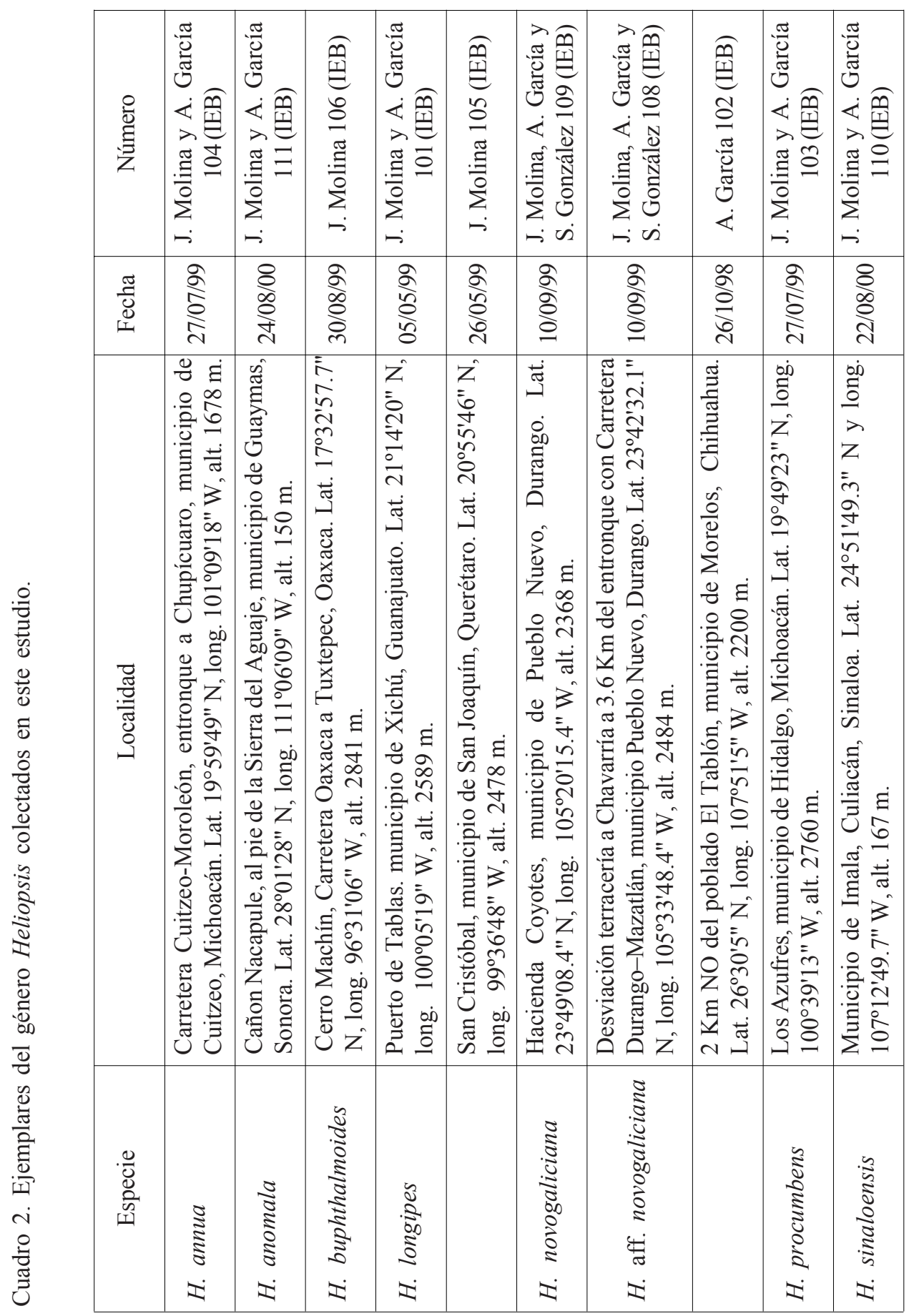


de separado el sobrenadante se evaporó el solvente con flujo de nitrógeno. El residuo fue resuspendido en un volumen de $500 \mu 1$ de etanol absoluto. Esta muestra se sometió a análisis por cromatografía de gases acoplada a espectrometría de masas (CG-MS) en las condiciones descritas previamente (Ramírez-Chávez et al., 2000). La caracterización de las alcamidas se hizo comparando el tiempo de retención en la columna y el espectro de masas de cada componente eluido. La estimación de la concentración de cada uno de estos compuestos se hizo sobre la base de una curva de calibración para la afinina ( $N$-isobutil-2E,6Z,8E-decatrienamida).

Resultados

De las siete especies colectadas sólo en seis se evaluó la presencia de alcamidas en raíces: $H$. longipes, $H$. procumbens, $H$. novogaliciana, $H$. annua, $H$. sinaloensis y $H$. buphthalmoides, siendo negativos los resultados para estas dos últimas especies. En el caso de H. anomala no se hizo la evaluación química debido a que sólo se logró la colecta de dos ejemplares, uno que se trató de conservar vivo para el establecimiento de cultivo de tejidos sin éxito, y el otro que fue herborizado. Se encontró un total de nueve alcamidas distintas, así como una estructura relacionada que es el 2E,6Z,8E-trien decanoato de bornilo (éster de bornilo), como se indica en el Cuadro 3. En cada columna para cada colecta se resalta con tipo más oscuro la alcamida dominante.

De las muestras analizadas, $H$. longipes es la especie con mayor cantidad de compuestos diferentes y contenido total de alcamidas. Los especímenes de las dos localidades donde se colectó, Puerto de Tablas, Guanajuato y San Cristóbal, Querétaro, mostraron algunas diferencias. El contenido más alto se observó en las muestras de Puerto de Tablas, con una concentración total de 9,369 $\mu \mathrm{g} / \mathrm{g}$ de peso seco de raíz repartido en ocho compuestos. A su vez, 4,592 $\mu \mathrm{g} / \mathrm{g}$ de peso seco de raíz en nueve compuestos, se obtuvieron de las raíces de plantas de San Cristóbal. En esta última localidad se observa la presencia de $N$-isobutil-2E,4Z,8Z,10E-tetraendodecamida, ausente en la primera, siendo en ambos casos la $N$-isobutil- $2 E, 6 Z, 8 E$ trien-decamida (afinina) la alcamida dominante con 7,827 y 3,968 $\mu \mathrm{g} / \mathrm{g}$ de peso seco de raíz respectivamente, seguida por la $N$-(2-metilbutil)-2E,6Z,8E-trien-decamida con 430 y $262 \mu \mathrm{g} / \mathrm{g}$ de peso seco de raíz. El resto de las alcamidas en forma minoritaria se conservan más o menos en la misma proporción en relación a la afinina para ambas localidades. En cuanto a la longitud de cadena, las decamidas son dominantes seguidas por undecamidas. En ambas localidades se detectó la existencia del éster de bornilo que, aunque no es una amida, su cadena acídica es igual a la de la afinina y por lo tanto su metabolismo está vinculado. La presencia de este compuesto sólo se conoce del género Heliopsis. 
García-Chávez et al.: Heliopsis en México y las alcamidas en sus raíces




Las alcamidas de $H$. novogaliciana difieren en forma importante en las localidades estudiadas. Las plantas de Hacienda Coyotes, Durango, despliegan cantidades muy bajas de amidas, únicamente afinina a nivel de trazas y la presencia de éster de bornilo ( $9 \mu \mathrm{g} / \mathrm{g}$ de peso seco de raíz), anteriormente sólo observado en $H$. longipes. Por otro lado, las plantas de $H$. aff. novogaliciana de las localidades de Chavarría en Durango y El Tablón en Chihuahua, muestran el perfil de los mismos siete compuestos en total, con $N$-isobutil- $2 E, 4 Z, 8 Z, 10 E$-tetraen-dodecamida como mayoritaria ( 177 y $594 \mu \mathrm{g} / \mathrm{g}$ de peso seco de raíz respectivamente), seguida de afinina (111 y $382 \mu \mathrm{g} / \mathrm{g}$ de peso seco de raíz respectivamente). En los especímenes de Chavarría y El Tablón, las dodecamidas son dominantes y no se observa el éster de bornilo.

Las raíces de $H$. procumbens presentan sólo dos alcamidas, ambas en baja concentración, siendo la dominante $N$-isobutil-2E,4Z,8Z,10E-tetraen-dodecamida (23 $\mu \mathrm{g} / \mathrm{g}$ de peso seco de raíz), seguida de afinina a nivel de trazas. En $H$. апnиa se observa solamente la presencia de afinina en baja concentración $(8 \mu \mathrm{g} / \mathrm{g}$ de peso seco de raíz), mientras que en $H$. buphthalmoides y $H$. sinaloensis no fue posible detectar este grupo de metabolitos en el intervalo de sensibilidad del equipo utilizado.

\section{Discusión}

Como era de esperarse, las raíces de $H$. longipes, el chilcuague, presentan el más alto contenido de afinina, lo que justifica su uso tradicional, que se destaca en ambas localidades de la Sierra Gorda. Es interesante mencionar que en el municipio de San Joaquín, Querétaro, y sus inmediaciones, no se reconoce la denominación de chilcuague, sino se denomina chilicuau a estas raíces, comercializándose en forma similar a su contraparte de los municipios de Xichú, San Luis de la Paz, Victoria y otros vecinos del estado de Guanajuato. Esta diferencia de nomenclatura sugiere que los habitantes que lo utilizan han tenido poco intercambio cultural a este nivel por un tiempo razonablemente largo.

Asímismo, se observan diferencias entre las plantas de estas dos localidades de la Sierra Gorda, geográficamente separadas por aproximadamente $60 \mathrm{~km}$ en línea recta. Ambos sitios están aislados por zonas de más baja altitud, menos húmedas y con condiciones que aparentemente no son propicias para el buen desarrollo de $H$. longipes. El contenido total de alcamidas es prácticamente el doble para los ejemplares de Guanajuato, lo que podría deberse a que la domesticación de la especie es mucho más marcada en esta región o a la diferencia de edad de las plantas estudiadas. La dodecamida con cuatro insaturaciones, $N$-isobutil-2E,4Z,8Z,10Etetraen-dodecamida detectada en la localidad de San Cristóbal, no se observó en trabajos previos sobre $H$. longipes, pues de la especie en cuestión no se conocían alcamidas de esta longitud de cadena (Molina-Torres et al., 1995). Los datos 
obtenidos sugieren que existen ya algunas diferencias genéticas entre las dos poblaciones mencionadas, lo que eventualmente podría llevar a la formación de variedades dentro de esta especie. En otros géneros de la tribu Heliantheae como Acmella, Echinacea o Salmea que contienen alcamidas, las de cadena dodeca son mayoritarias. Heliopsis longipes es la única especie conocida que presenta alcamidas olefínicas solamente de diez carbonos, con la excepción mencionada de la localidad de San Cristóbal.

Adicionalmente, el éster de bornilo se incluye en este estudio por estar constituido por la misma cadena alifática del ácido $2 E, 6 Z, 8 E$-trien-oico de las alcamidas mayoritarias de las especies de Heliopsis. Este compuesto está presente en los especímenes de ambas localidades de H. longipes de la Sierra Gorda, fue descrito inicialmente en esta especie (Molina-Torres et al., 1995) y ahora se registra para $H$. novogaliciana. Hasta la fecha no se ha encontrado fuera de este género. En cuanto a las plantas identificadas como de $H$. novogaliciana y $H$. aff. novogaliciana, existen diferencias importantes en el perfil de alcamidas presentes en sus raíces entre la primera y la segunda. Los ejemplares colectados en Hacienda Coyotes en Durango, contienen sólo pequeñas cantidades de dos de los compuestos en estudio: la afinina y el éster de bornilo. Los especímenes de $H$. aff. novogaliciana de las localidades de Chavarría en Durango y El Tablón en Chihuahua, contienen las mismas siete alcamidas, siendo dominantes las dodecamidas tetrainsaturadas. Aunque el material de Chihuahua contiene una cantidad total de alcamidas tres veces mayor que el de Durango (1,109 vs $364 \mu \mathrm{g} / \mathrm{g}$ de peso seco de raíz), no se puede descartar la posibilidad de que las muestras analizadas sean de plantas de diferente edad. Este punto de colecta, El Tablón en Chihuahua, es un lugar relativamente aislado y alejado de sitios registrados previamente para este género.

Por último, las raíces de las otras cuatro especies analizadas (H. procumbens, $H$. апnиа, $H$. sinaloensis y $H$. buphthalmoides) sólo revelan niveles de alcamidas con valores totales inferiores a $25 \mu \mathrm{g} / \mathrm{g}$ de peso seco de raíz. H. procumbens presenta solamente $24 \mu \mathrm{g} / \mathrm{g}$ de la dodecamida que predomina en las $H$. aff. novogaliciana, mientras que $H$. апnиa contiene únicamente $8 \mu \mathrm{g} / \mathrm{g}$ de peso seco y las otras dos muestran niveles no evaluables de estos metabolitos. Tomando en cuenta la cantidad de alcamidas en las raíces de las plantas estudiadas, se les puede subdividir tentativamente en dos grupos: aquellas especies con altos niveles y diversidad de alcamidas, que incluye a $H$. longipes y a $H$. aff. novogaliciana de las localidades de Chavarría y El Tablón; y un segundo grupo con bajas cantidades y variedad de estos compuestos en el que quedan incorporadas: H. novogaliciana, $H$. procumbens, $H$. annua, $H$. sinaloensis y $H$. buphthalmoides.

Los datos hasta aquí presentados muestran diferencia significativa entre los diferentes taxa en el género Heliopsis y despiertan algunas dudas, por lo que se advierte la necesidad de ampliar el estudio, así como explorar el uso de nuevas 
estrategias de investigación, como las diferentes técnicas fundamentadas en las secuencia del ADN. Actualmente se desarrolla un estudio con marcadores moleculares sobre la base de RAPDs e ITSs tendientes a robustecer la correcta ubicación taxonómica de estas especies.

\section{AGRADECIMIENTOS}

Los autores desean agradecer al Dr. Jerzy Rzedowski, por la ayuda en la identificación de especies; a la Dra. Socorro González Elizondo por el apoyo en el trabajo de campo y la revisión de un manuscrito preliminar; a CONACYT convenio: 26398N, Sistema de Investigación Miguel Hidalgo (SIHGO) convenio 97810 y Fundación Guanajuato Produce convenio 31/98, por el soporte económico para la realización de esta contribución.

\section{LITERATURA CITADA}

Acree, F., M. Jacobson y H. L. Haller. 1945. The structure of affinin, the insecticidal amide from Erigeron affinis D.C. Journal of Organic Chemistry 10: 449-451.

Blake, S. 1924. New American Asteraceae. Heliopsis longipes (A. Gray) Blake. Contributions from the U.S. National Herbarium 22: 608-609.

Bremer, K. 1994. Asteraceae: cladistics \& classification. Timber Press. Portland. 752 pp.

Christensen, L. 1992. Acetylenes and related compounds in Anthemideae. Phytochemistry 31: 7-49.

Christensen, L. y L. Lam. 1991. Acetylenes and related compounds in Heliantheae. Phytochemistry 30: 11-49.

Díaz-Piedrahíta, S. 1990. Acerca de la validez de dos nombres de asteráceas colombianas y su correcta tipificación. Revista de la Academia Colombiana de Ciencias Exactas, Físicas y Naturales 67: 645-648

Fisher, T. R. 1954. A new species of Heliopsis from Baja California, Mexico. Madroño 12: $152-155$.

Fisher, T. R. 1957. Taxonomy of the genus Heliopsis (Compositae). Ohio Journal of Science 57: 171-191.

Fisher, T. R. 1961. A new species of Heliopsis from Mexico. Ohio Journal of Science 61: 178-179.

Gray, A. 1879. Characteristics of some new species of Compositae. Proceedings of the National Academy of Sciences, USA 15: 25-51.

Greger, H. 1984. Alkamides: structural relationships, distribution and biological activity. Planta Medica 50: 366-375.

Gutiérrez-Lugo, M. T., T. Barrientos-Benitez, B. Luna, R. M. Ramírez-Gama, R. Bye, E. Linares y R. Mata 1996. Antimicrobial and cytotoxic activities of some crude drug extracts from Mexican medicinal plants. Phytomedicine 2(4): 341-347. 
Jacobson, M. 1951. Constituents of Heliopsis species. I. Scabrin, an insecticidal amide from the roots of H. scabra Dunal. Journal of the American Chemical Society 73: 100-103.

Little, E. 1948. El chilcuague (Heliopsis longipes). Planta insecticida. Boletín de la Sociedad Botánica de México 7: 23-27.

Martínez, M. 1933. Las plantas medicinales de México. 6a ed. Editorial Botas. México D.F. $656 \mathrm{pp}$.

McVaugh, R. 1984. Heliopsis Pers. In: Flora Novo-Galiciana. Compositae. 12: 496-502.

Molina-Torres, J., R. Salgado-Garciglia, E. Ramírez-Chávez y R. del-Rio. 1995. Presence of the bornyl ester of deca-2E,6Z,8E-trienoic acid in Heliopsis longipes roots. Journal of Natural Products 58: 1590-1591.

Molina-Torres, J., R. Salgado-Garciglia, E. Ramírez-Chávez y R. del-Rio. 1996. Purely olefinic alkamides in Heliopsis longipes and Acmella (Spilanthes) oppositifolia. Biochemical Systematics and Ecology 24: 43-47.

Molina-Torres, J., A. García-Chávez y E. Ramírez-Chávez. 1999. Antimicrobial properties of natural alkamides traditionally used in Mesoamerica: affinin and capsaicin. Journal of Ethnopharmacology 64: 241-248.

Noriega, J. 1902. Curso de historia de drogas. Instituto Médico Nacional. Oficina Tipográfica de la Secretaría de Fomento, México, D.F. 837 pp.

Parmar, V. S., S. C. Jain, K. S. Bisht, R. Jain, P. Taneja, A. Jha, O. D. Tyagi, A. K. Prasad, J. Wengel, C.E. Olsen y P.M. Bool 1997. Phytochemistry of the genus Piper. Phytochemistry 46: 597-673.

Ramírez-Chávez, E., L. Lucas-Valdez, G. Virgen-Calleros y J. Molina-Torres. 2000. Actividad fungicida de afinina y extracto crudo de raíces de Heliopsis longipes sobre dos especies de Sclerotium. Agrociencia 34: 207-217.

Roark, R.C. 1951. Scabrin. Soaps and sanitary chemicals 27: 125,137.

Romero, C. M., A. R. Del-Castillo, A. C. Martínez y C. J. Calderón. 1989. Preliminary studies on the antibacterial, insecticidal and toxicological effects of the chilcuan root Heliopsis longipes. Veterinaria 20: 151-156.

Rzedowski, G. C. de, J. Rzedowski y colaboradores. 2001. Flora fanerogámica del Valle de México. 2a ed., Instituto de Ecología, A.C. y Comisión Nacional para el Conocimiento y Uso de la Biodiversidad, Pátzcuaro. 1406 pp.

Turner, B. L. 1987. Two new species of Heliopsis (Asteraceae) from northwestern Mexico. Phytologia 63: 1-3.

Turner, B. L. 1988. Comments upon and new combinations in Heliopsis (Asteraceae, Heliantheae). Phytologia 64: 337-339.

Wilson, P. G. 1958. Contributions to the flora of tropical America: LXIII. Kew Bulletin 164: $155-165$.

Ximénez, F. 1615. Qvatro libros de la natvraleza. Edición facsimilar impresa en Morelia, 1888. $300 \mathrm{pp}$.

Recibido en octubre de 2001 Aceptado en noviembre de 2004 